\title{
S-Parameter Comparison of Common Source and Common Gate Low Noise Amplifier
}

\author{
Mohit Dayal \\ M.Tech Student \\ Lovely Professional University
}

\author{
Abhishek Kumar \\ Assistant Professor \\ Lovely Professional University
}

\begin{abstract}
Scattering Parameters or S-parameters are complex numbers which tells us about how voltage waves are propagating in the radio-frequency (RF) environment. In this paper common source and other will be the cascoded common gate topologies have been analyzed. Scattering parameter analysis shows that gain and impedance matching is better for CG LNA. S-parameter is an important tool for LNA design, following information can be obtained a) The frequency b) characteristic impedance (often $50 \Omega$ ) c) The allocation of port numbers d)Operating environment such as temperature, control voltage and bias current.
\end{abstract}

\section{Keywords}

LNA, Radio Frequency, Gain, Scattering parameters.

\section{INTRODUCTION}

An LNA is a key component used in communication system. LNA is placed right to antenna (detection device) in the receiver side. LNA amplifies very weak signals captured by an antenna. Role of LNA is to amplify the signal shown in fig 1. Quality of signal degrades due to availability of noise in environment and electronic component.

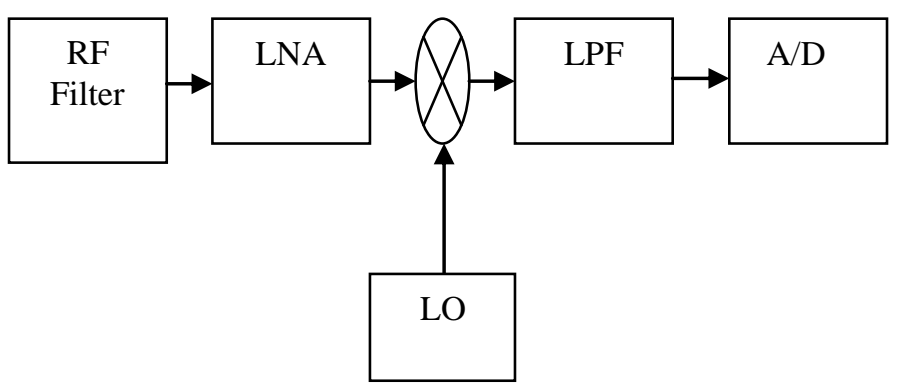

Fig.1. RF Receiver

Fig 2 shows a normal amplifier has gain A1 amplifies the signal as well as input noise present.

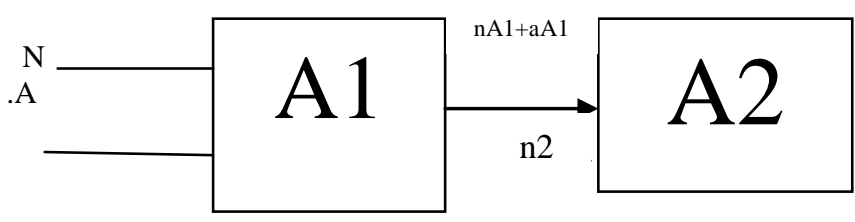

Fig2. Amplifiers in series

$\mathrm{S} / \mathrm{N}$ at the input is given as a/n. Noise is amplified by factor $\mathrm{A} 1$, another noise component $\mathrm{n} 2$ get added due to subsequent stage. SNR at input of $2^{\text {nd }}$ stage $=a A 1 /(n A 1+n 2)$ if gain of $1^{\text {st }}$ stage is very large.

$$
\mathrm{SNR}=\mathrm{a} / \mathrm{n}
$$

It shows if gain if $1^{\text {st }}$ stage is large than noise of $1^{\text {st }}$ stage is important, subsequent stage noise is irrelevant because subsequent stage adds small quantity of noise while $1^{\text {st }}$ stage already has amplified noise, So subsequent system noise can be ignored. A low noise amplifier does not add much noise but amplify. An LNA add very low noise by own but amplify everything as shown in fig3.

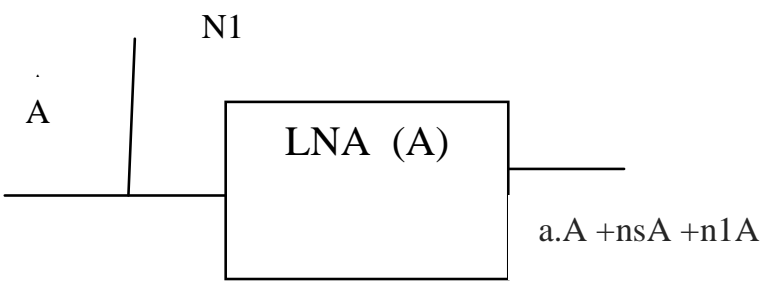

Fig3. Low noise amplifier Noise Analysis

$$
\mathrm{SNR}=\text { a.A } / \quad(\text { n1.A+ns.A })
$$

$\mathrm{n} 1=$ input referred noise

ns $=$ noise source

$\mathrm{a}=$ signal

The four most important parameters in LNA design are:

1. Gain Should Be High

2. Noise Figure NF Should Be Low

3. Non-Linearity

4. Impedance Matching: Input And Output Impedance Should Be Matched

Impedance Matching:- Input impedance of LNA should be match with impedance of antenna. Impedance matching is required for better reception (shape) of signal. If impedance is not matched signal get distorted. Also, maximum power transfer theorem says maximum power will be transfers if source impedance is conjugate of input impedance. Similarly output impedance of LNA should be matched with subsequent stage to conserve shape of signal .

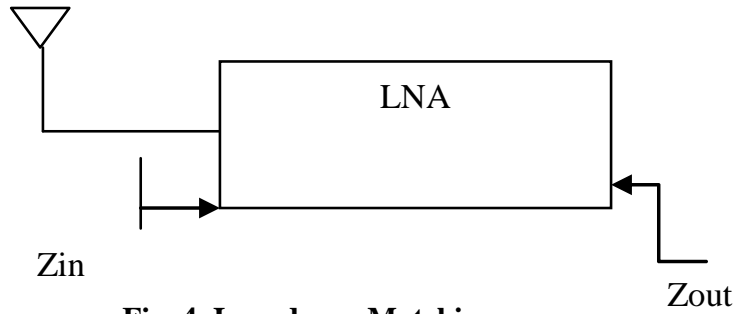

Fig. 4. Impedance Matching
Zout 
As in this Fig 4 Zin and Zout are two Impedances on input and output respectively and there values should be matched accordingly. As Rs should be equal to Zin and Zout value should be equal to next stage impedance.

\section{SCATTERING PARAMETERS}

Scattering Parameters or S-parameters are complex numbers which tells how voltage waves are propagating in the radiofrequency (RF) environment. They characterize the complete RF behavior of a network in Matrix form. S-parameter used to represent 2-port electrical equivalent circuit of $\mathrm{RF} /$ microwave. For a two-port device there are four $\mathrm{S}$ parameters S11, S21, S12, and S22. S11 and S22 are simply the forward and reverse reflection coefficients, with the opposite port terminated in Z0 (usually $50 \mathrm{ohms}$.) S21 and S12are forward and reverse gain.

The properties of the individual components and those of the physical structure of the circuit are effectively derived from the equation and circuit analysis is greatly simplified. The characteristics of the 2-port is represented by a set of four Sparameters[1,2]:

\section{S11: Input Reflection Coefficient}

\section{S12: Reverse Gain Coefficient}

\section{S21: Forward Gain Coefficient}

\section{S22: Output Reflection Coefficient}

S-parameters are measured under matched and mismatched conditions. This is why S-parameters are favored in microwave applications. S-parameters are both frequencyand system impedance dependent so although manufacturers typically supply S-parameter data with their devices it is not always applicable. Under such circumstances, it becomes necessary to measure the parameters.

We can characterize the behavior of Two port network with various methods. In case when frequency is low we can use parameters like $\mathrm{Y}, \mathrm{Z}, \mathrm{H}, \mathrm{T}$ and $\mathrm{ABCD}$. These parameters works under open and short circuit conditions to give information about a linear electric network. But these parameters are tough to realize in case of high frequency models. In radio frequency range, scattering parameters ( $\mathrm{S}$ Parameters) is normally employed. It uses matched load termination and the measurements are based on incident and reflected waves [3].

Fig5 shows a two-port network, where $\mathrm{a}_{1}$ and $\mathrm{a}_{2}$ are incident waves; $b_{1}$ and $b_{2}$ are reflected waves. Their relation is expressed as[2]:

$$
\left[\begin{array}{l}
b_{1} \\
b_{2}
\end{array}\right]=\left[\begin{array}{ll}
S_{11} & S_{12} \\
S_{21} & S_{22}
\end{array}\right]\left[\begin{array}{l}
a_{1} \\
a_{2}
\end{array}\right]=[S]\left[\begin{array}{l}
a_{1} \\
a_{2}
\end{array}\right]
$$

The $[\mathrm{S}]$ matrix is called scattering matrix.

$$
\begin{aligned}
& \mathrm{S}_{11}=\frac{\mathrm{b}_{1}}{\mathrm{a}_{1}} \mid \mathrm{a}_{2}=0 \text { With matched output port } \\
& \mathrm{S}_{12}=\frac{b_{1}}{a_{2}} \mid \mathrm{a}_{1}=0 \text { With matched input port } \\
& \mathrm{S}_{21}=\frac{b_{2}}{a_{1}} \mid \mathrm{a}_{2}=0 \text { With matched output Port } \\
& \mathrm{S}_{22}=\frac{b_{2}}{a_{2}} \mid \mathrm{a}_{1}=0 \text { With matched input port }
\end{aligned}
$$

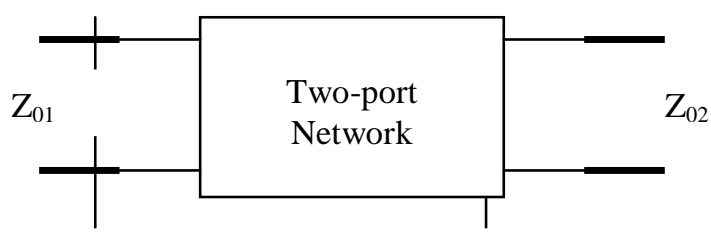

Fig 5. Two Port Network
Conclusively, the S-parameters relate the four waves in the following fashion[1,2]:

$$
\begin{gathered}
b_{1}=S_{11} a_{1}+S_{12} a_{2} \\
b_{2}=S_{21} a_{1}+S_{22} a_{2}
\end{gathered}
$$

\section{S-PARAMETER ANALYSIS}

The S-Parameter analysis is used to compute scattering and noise parameters for 2-port circuits that exhibit frequency translation. S-Parameter analysis is used for small-signal and linear noise analyses, where the circuits are linearized around the DC operating point. Such circuits include LNAs, mixers, samplers and other similar circuits. Cadene spectre used to do SP analysis and noise parameters in frequency-converting circuits. SP computes noise figure, input referred noise, equivalent noise parameters, and noise correlation matrices. For the SP analysis, it is required to specify the input and output ports and the range of sweep frequencies. SP analysis for the LNA provides gain and matching parameters measurements [4].

1. S11, input reflection coefficient

2. S21, forward gain

3. S12, reverse gain

4. S22, output reflection coefficient

5. GT, transducer power gain

6. GP, operating power gain

7. GA, available power gain

By setting the input and output noise port, I can plot NF and $\mathrm{NF}_{\min }$ with respect to frequency. The Stern stability factor $\mathrm{K}_{\mathrm{f}}$ and $\Delta$ can be plotted with respect to frequency.

\begin{abstract}
S-Parameter analysis of Common Source Low Noise Amplifier:

The Common Source topology is most basic one in case of LNA topologies. Main reason for choosing this topology was it can give high voltage gain and that's the main requirement in case of LNAs. Apart from high gain it comes with some problems like high output impedance. Though its not as such big issue as compare to its main drawback which is limited high frequency response and it because of presence of $C_{g d}$ i.e. capacitance present between the gate and drain terminal in MOS[5]. we can see this in the small signal circuit model of Fig7. As we are saying its having high gain as we can see in value of $S_{21}$ but its value is negative near to $22 \mathrm{~dB}$. Because as basic common source is having phase shift of the 180 and it will completely turn value into opposite phase Thus we will be having negative value of the $S_{21}$.
\end{abstract}

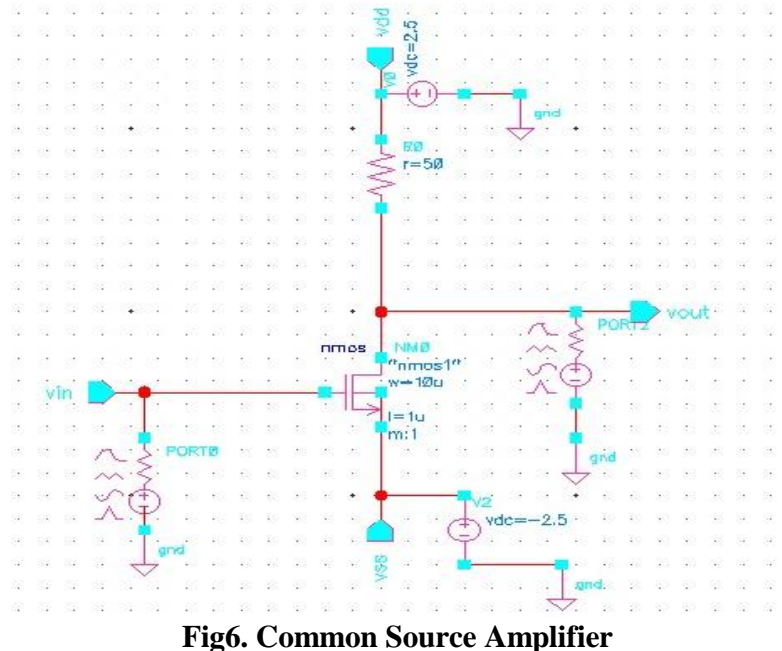

Fig6. Common Source Amplifier 
To avoid the problem of capacitance we can use Miller's Theorem, means we will split the capacitance $C g d$ into two capacitances given by at the $C_{g d}$. $\left(1-A_{v}\right)$ value at the input and $C_{g d} \cdot\left(1-\frac{1}{A_{v}}\right)$ value at the output, in these expressions $A_{v}$ is the voltage gain of the amplifier [6].as we said gain will be high and negative then capacitance between gate and source will become very large also and it will result in a low high frequency pole given by

$$
p_{1}=\frac{1}{R_{i n}+\left(C_{g s}+C_{g d} A_{v)}\right.}
$$

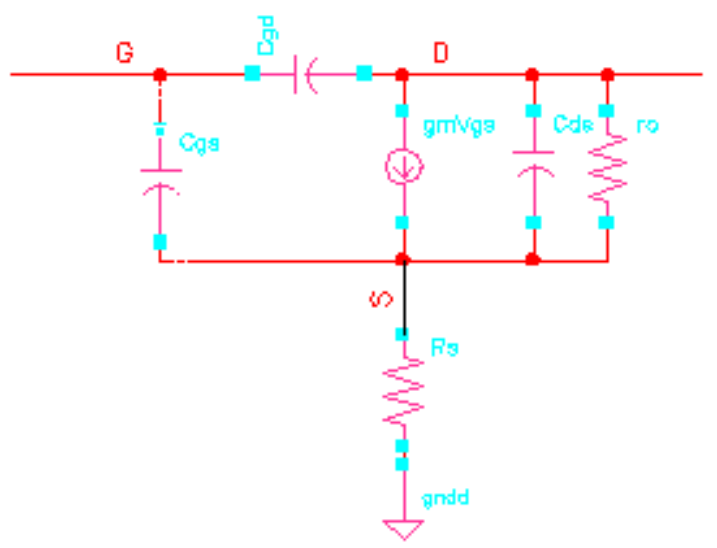

Fig7. Small signal Model of Common Source

Gain is decreasing along with frequency as we told earlier due to high frequencies there is large effect of miller effect and that leads to inefficiency of CS amplifier to work on High Frequency. In order to make this circuit work on high frequency at least near to $900 \mathrm{MHz}$ this circuit was modified and in this modified circuit effect of these characteristics on the performance was less. Simulation result of common source s parameter shown in fig 8

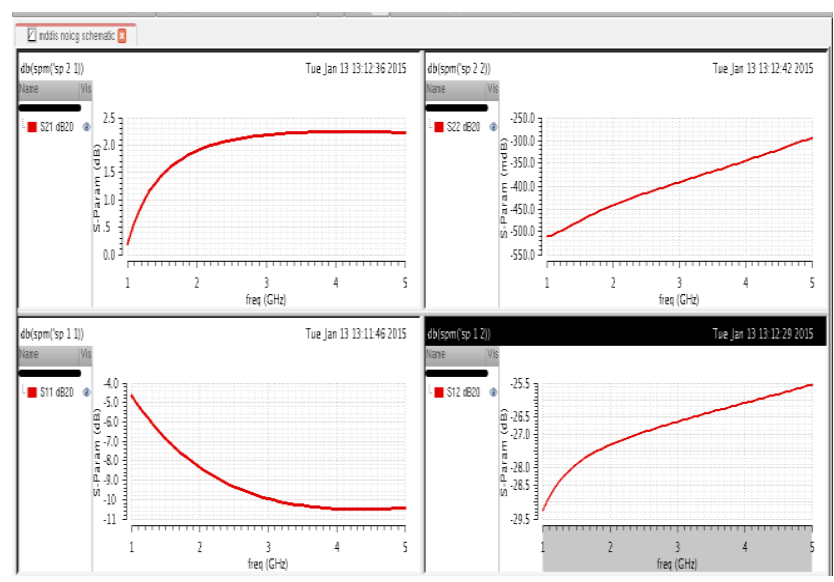

Fig8. S-parameter Analysis of Common Source

$S$ parameter analysis of Cascoded Common gate with low noise:

The parallel RLC input matching network of the CGLNA limits its noise and gain performance. At resonance, the CGLNA's input impedance is $1 / \mathrm{g}_{\mathrm{m}}$. We wished to improve the NF of the Common Gate amplifier using shunt inductor. As reported NF of conventional basic Common Gate amplifier is nearly $5 \mathrm{~dB}$, with this design we will be getting noise figure of 2.6dB.On-chip inductor $L_{d}$ acts as output impedance. Capacitors $\mathrm{C}_{\mathrm{b}}$ are bypass capacitors. On-chip inductors $\mathrm{L}_{\mathrm{s}}$ are used to tune out the total capacitances at the source nodes including pad capacitances[7]. The second stage of the LNA acts as an output buffer. It has $50 \Omega$ output impedance to match with the $50 \Omega$ load of the measuring equipment.

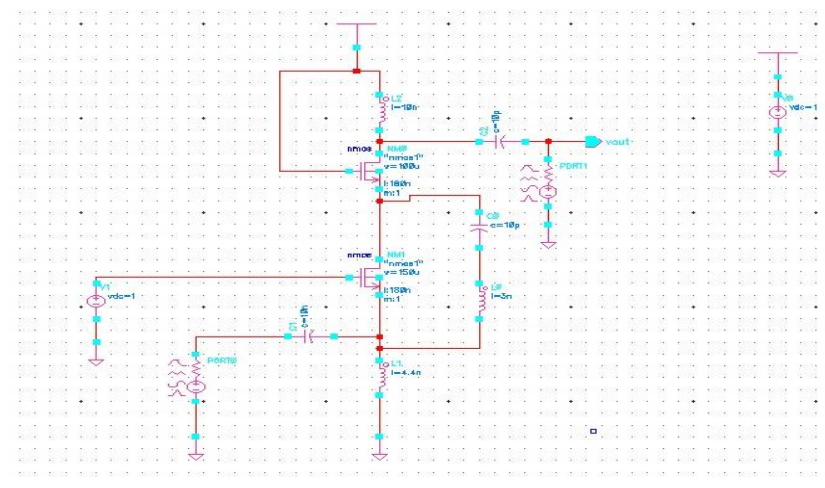

Fig9. Cascoded Common Gate schematic

Circuit given in Figure 9 is proposed for better noise figure performance of the common gate using cascoded circuit as well as $\mathrm{C}_{\mathrm{b}}$ and $\mathrm{L}_{\mathrm{a}}$ are used to control the noise figure. With the help of small signal analysis we can calculate different values of our capacitors and inductors and then we will simulate that circuit in the Cadence to check the values. Simulation result of common source s parameter shown in fig 10 .

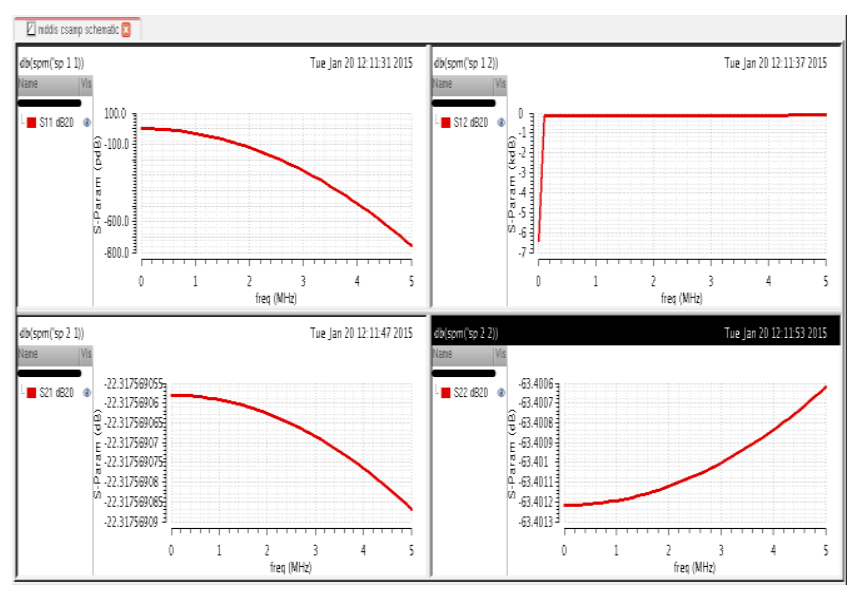

Fig10. S-parameter Analysis of cascoded Common Gate

\section{CONCLUSION}

The LNA is designed in CMOS 180nm technology. Common source amplifier is having fewer values and moreover values are not optimum due to working of basic common source amplifier, on the other hand results of Cascoded Common gate is very much on the required side as $S_{21}$ is giving reasonable gain as circuit is much dependent on noise reduction. $S_{11}$ and $S_{22}$ are providing good values for input and output matching as compare to the common source amplifier. $\mathrm{S}_{12}$ is providing better reverse isolation rather than that of common source amplifier. 
TABLE I

\begin{tabular}{|l|l|l|}
\hline S-Parameter & $\begin{array}{l}\text { Common Source } \\
\text { Topology }\end{array}$ & $\begin{array}{l}\text { Cascoded } \\
\text { Common Gate } \\
\text { Topology }\end{array}$ \\
\hline$S_{11}$ & $-150 \mathrm{pdB}$ & $-10 \mathrm{~dB}$ \\
\hline $\mathrm{S}_{12}$ & $-1 \mathrm{kdB}$ & $-27 \mathrm{~dB}$ \\
\hline $\mathrm{S}_{21}$ & $-22 \mathrm{~dB}$ & $2.5 \mathrm{~dB}$ \\
\hline $\mathrm{S}_{22}$ & $-63 \mathrm{~dB}$ & $-.45 \mathrm{~dB}$ \\
\hline
\end{tabular}

Result for $\mathrm{S} 11$ is $-10 \mathrm{~dB}$ and the frequencies are at $2.4 \mathrm{GHz}$ while S21 is observed $2.5 \mathrm{~dB}$ and these values show that the resistance at the input of LNA is matched. In the end if we want to look at future, these LNA's can be modified according to user's requirements and in communication purposes they have a highly rated future.

\section{REFERENCES}

[1] Prof. L. Dunleavy "S-parameters MUSE" University of South Florida.

[2] M.Muhmad and N. A.Nordin, "S-parameter analysis of 0.18um LNA for WCDMA application" Proceedings of the 9th WSEAS International Conference on Microelectronics and oproelectronics,2004.

[3] Srinivas Jagarapu and R.K. Kavitha "A $2.4 \mathrm{GHz}$ Low Noise Amplifier in 0.18um CMOS Technology" Proceedings of International Journal of Computer Applications ${ }^{\circledR}$ (IJCA) 2011.

[4] Thomas H. Lee, "The Design of CMOS RadioFrequency Integrated Circuit," Cambridge University Press, 1998.

[5] Anderson, R. W., "S-parameter techniques for faster, more accurate network design," Hewlett-Packard Application Note 95-1, 5952-1130, 1997.

[6] Anon., "S Parameter Design," Agilent Technologies application note, AN 154,2006

[7] Hong Qi and Zhang Jie, "A 1.5V Low Power CMOS LNA Design," International Symposium on Microwave, Antenna, Propagation and EMC Technologies for Wireless Communications, pp. 1379 - 1382 , Aug. 2007. 